LABORATORY MEDICINE

\title{
Blood glucose monitors: Technology for home healthcare
}

WALTER C. HOOK, MT(ASCP), MS

JOHN FERNANDES, DO

Blood glucose self-monitoring offers several advantages. This article, the first in a series, focuses on the types of monitors currently marketed and their limitations. Because optimal patient care rests with operator training, this article also places emphasis on the need to properly train those who will be performing blood glucose monitoring.

(Key words: Blood glucose monitors, glucose monitors, self-monitoring, home healthcare, devices)

Blood glucose self-monitoring is an accepted routine in the management of diabetes mellitus. Blood glucose monitors (BGMs) are relatively inexpensive devices, provide reliable glucose quantification, and are easy to operate. Consequently, blood glucose testing that was formerly performed only in clinical laboratories can now be performed at a patient's bedside in an institutional setting as well as in the home. The primary advantage of blood glucose monitoring is that the patient has greater responsibility for monitoring therapy. Because renal function and glucose clearance may change significantly during the course of diabetes, blood glucose concentrations better represent the patient's glycemic state than do urine glucose measurements. Microvascular

From the Department of Pathology and Laboratory Medicine, Philadelphia College of Osteopathic Medicine, Philadelphia, $\mathrm{Pa}$.

Reprint requests to John Fernandes, DO, Chairman, Department of Pathology and Laboratory Medicine, Philadelphia College of Osteopathic Medicine, 4150 City Ave, Philadelphia, PA 19131. and neurologic complications may be reduced in diabetic patients who are in better control of their glucose concentrations. This management is significantly aided by glucose selfmonitoring. Noninsulin-dependent diabetics can also use self-monitoring to modify their diets to maintain glucose control.

The use of BGMs gives the patient greater freedom. The patient is no longer tied to the hospital laboratory for glucose measurements. Self-monitoring can be performed at any time in any location without disrupting the active lifestyle of many patients.

Currently available BGMs (Table) use either reflectance photometry or electrochemistry to quantify glucose.

\section{Reflectance photometry}

To monitor blood glucose by use of reflectance photometry, a drop of blood (usually obtained from a finger stick or earlobe puncture) is placed onto a reagent-impregnated test pad or film that is supported on a thin plastic strip. The drop of blood is allowed to incubate for a prescribed time, and wiped off. During the incubation time, serum diffuses into the pad and reacts with glucose oxidase and other reagents to produce a color. The intensity of the color is proportional to the concentration of glucose in the blood. The strip is inserted into the monitor. A diode emits light at a specific wavelength, which is reflected off the reacted reagent film. A photodetector monitors the reflected light and converts it into an electric signal that is processed and transformed into a number that represents the glucose concen- 
tration in the blood. The glucose concentration then is displayed digitally on the monitor.

All reflectance BGMs except the LifeScan One Touch require the operator to monitor the incubation of the blood on the test film, to wipe the blood off the film at a specified time, and to insert the strip into the monitor to obtain a glucose measurement. In using the One Touch, the operator places a test strip into the instrument, and then turns on the instrument. A light-emitting diode flashes light against the white surface of the test strip. If the strip is properly inserted, and the reagents are stable, the instrument then signals the operator to apply a drop of blood to the test pad. The One Touch automatically initiates the timing sequence when the serum from the drop of blood has diffused through the test pad, and displays the blood glucose concentration within 45 seconds.

\section{Electrochemical blood glucose monitors}

Three electrochemical blood glucose monitors are currently available: the Exactech, the Exactech Companion, and the Direct 30/30.

To measure glucose with the Exactech, the operator inserts a test strip into the pen-sized monitor. The strip contains electrodes that pass through the reagents, which impregnate the outer portion of the strip. The operator must place a drop of blood onto the reagent area and simultaneously depress a button at the opposite end of the device to start a timing sequence. At the end of 30 seconds, the instrument measures the flow of electrons generated during the chemical reaction of glucose with reagents in the test strip and digitally displays the glucose concentration.

The Direct $30 / 30$ operates on a similar electrochemical principle. However, with this device, the reagent impregnates a sensor that remains in the instrument and does not have to be replaced after each use. The sensor is stable for 30 days.

\section{Quality of measurements}

The major factors that influence the quality of blood glucose measurements made with a BGM are:
- insufficient blood on the test strip;

- hematocrit value $<30 \%$ or $>50 \%$;

- underincubation or overincubation of glucose on the test strip;

- poor wiping or blotting technique;

- chemical interference; and

- insufficient operator training.

\section{Insufficient specimen}

Capillary blood is the specimen of choice for most blood glucose monitors. The specimen can be obtained from a finger or earlobe puncture, although finger sticks are most commonly used. The first drop of blood should be discarded, and the next drop of blood is placed on the test strip. It is imperative that the blood sample be sufficient to cover the test-pad portion of the strip for two reasons: (1) the photooptical systems of BGMs that use reflectance photometry are designed to scan the entire test pad. Therefore, if there were insufficient sample on the strip, portions of the test pad would not have color changes that result from the reaction of glucose with reagents in the pad. The BGM readout would be incorrect. (2) Electrochemical BGMs require that the operator place a sufficient blood sample onto the reagent pad to allow the reaction of glucose in the sample to proceed optimally. Too little sample would yield lower-than-actual blood glucose readings.

\section{Hematocrit}

Blood glucose monitors are specifically designed, and factory-calibrated, to measure capillary whole blood glucose. (The Diascan S may be set by the operator to measure glucose in capillary or venous whole blood.) The glucose concentration in whole blood differs from that measured in plasma or serum. Furthermore, fingerstick whole blood is obtained from capillaries. The concentration of glucose decreases as blood flows from arteries to capillaries to veins. Therefore, blood from a finger stick will have a higher glucose concentration than venous blood, the specimen of choice for larger laboratory-based chemistry analyzers. However, typically blood glucose concentrations are lower on most BGMs that use reflectance photometry than the concentrations reported from 


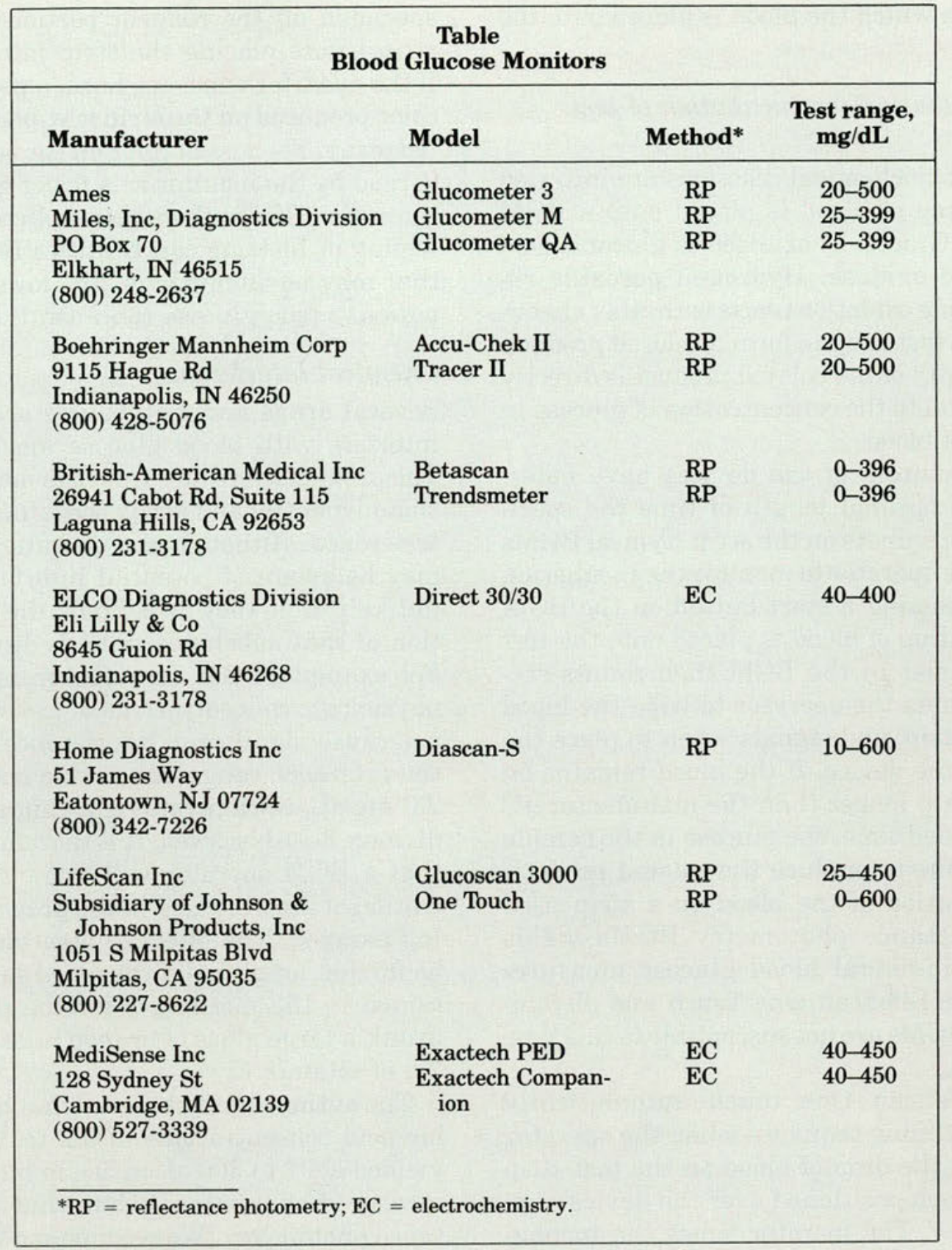

the same patient when simultaneously collected venous plasma or serum is measured in the laboratory.

When whole blood is placed on the test strip, the red blood cells occupy "dead space" that reduces the surface area of reagent that is exposed to the serum. As a result, the concentration of glucose that comes into contact with the reagent in the pad is influenced by the volume of red blood cells in the specimen-the hematocrit. The manufacturers of BGMs that use reflectance photometry are aware of this phenomenon and have optimized their BGMs to measure primarily between the hematocrit range of $30 \%$ to $50 \%$.

The LifeScan One Touch is the least susceptible of all monitors to hematocrit variations because the fluid portion of the blood diffuses through the test pad. The instrument scans the reaction on the test strip opposite 
the side on which the blood is placed onto the strip.

\section{Underincubation/overincubation of test strip}

A series of biochemical reactions are initiated when a drop of blood is placed onto a BGM test strip. Glucose is oxidized to gluconic acid by glucose oxidase. Hydrogen peroxide released during oxidation reacts with other chemicals in the test strip to form a colored product. The intensity of the colored product is directly proportional to the concentration of glucose in the drop of blood.

Manufacturers of the devices have determined the optimal length of time the specimen must incubate on the strip. Typical BGMs require the operator to monitor the incubation time by pressing a start button on the BGM when the drop of blood is placed onto the test strip. A timer in the BGM then counts seconds, prompts the operator to-wipe the blood from the strip, and signals when to place the strip into the device. If the blood remains on the test strip longer than the manufacturer's recommended time, the glucose in the sample will continue to produce the colored product. Overincubation of the blood on a strip used with reflectance photometry BGMs yields higher-than-actual blood glucose measurements. The LifeScan One Touch and electrochemical BGMs are not susceptible to this type of problem.

The LifeScan One touch automatically starts the timing sequence when the operator has placed the drop of blood on the test strip that has been positioned over the device's optics window. The monitor times the incubation and automatically reads the test result without any other operator intervention. The Exactech requires the operator to initiate the incubation timing sequence when the drop of blood is placed onto the test strip, but requires no further operator intervention to obtain the test result.

\section{Improper wiping technique}

As aforenoted, all BGMs, with the exception of the One Touch, Exactech, and Direct 30/30, require the operator to wipe or to blot the blood specimen off the reagent portion of the test strip before placing the strip into the BGM. If the operator wipes too hard, a portion of the color produced on the strip is wiped off or blotted away. The loss of color on the reacted strip is read by the monitor as a lower glucose concentration. The authors have noticed that overwiping or blotting can result in BGM results that may be as much as $20 \%$ lower than the patient's true glucose concentration.

\section{Chemical interference}

Several drugs and metabolites are known to interfere with blood glucose measurements. The product literature from the manufacturer should identify and verify the effects of the interference. Although the operators of BGMs may be aware of potential interference, it is unlikely that they will know the concentration of that substance in each drop of blood. For example, BGM manufacturers state that physiologic concentrations of ascorbic acid do not cause significant interference. Although the reference range of ascorbic acid is 0.2 to $2.0 \mathrm{mg} / \mathrm{dL}$, concentrations as high as $50 \mathrm{mg} /$ $\mathrm{dL}$ have been observed. It is therefore unlikely that a BGM operator will know the concentration of ascorbic acid in the drop of blood being assayed. The effect of ascorbic acid may be further amplified if the blood sample is obtained in the morning after the patient has drunk a large glass of orange juice and taken $2 \mathrm{~g}$ of vitamin C.

The authors have observed that blood ascorbic acid concentrations of 0.2 to $16.9 \mathrm{mg} / \mathrm{dL}$ yielded a $5 \%$ to $30 \%$ decrease in blood glucose measured on several BGMs that use reflectance photometry. We recommend that BGMs not be used to quantify glucose in patients who take large doses of ascorbic acid or who are receiving megavitamin therapy. (Larger laboratory analyzers, although not immune, are less susceptible to this interference because of their technology.)

\section{Human factors, ease of use, and operator training}

In the experience of one of the author's (W.C.H.), there were significant differences in test results obtained by trained operators when 
compared with those results obtained by untrained users. The differences in glucose measurements were traced primarily to human factors that involve ease of use. Instruments that are easier to operate provide the best results. The quality of results is better on instruments that allow the operator to easily perform the test without additional specimen and reagent processing than on those that require operators to time incubation and wipe blood from test strips.

However, regardless of how easy the BGM is to operate, there is no substitute for proper operator training. The adage that "practice makes perfect" is not true when using BGMs. Proper training and proper practice make perfect. If the operator is inadequately trained, repetition of the incorrect practice will reinforce bad habits and perpetuate errors in blood glucose monitoring.

Blood glucose monitors have given patients, physicians, and allied health professionals the ability to rapidly, and accurately, detect and monitor fluctuations in blood glucose. The accuracy of results depends greatly on operator technique. With many monitors, one of the authors (W.C.H.) has observed blood glucose concentrations that were greater than $25 \%$ lower than reference glucose values obtained by use of clinical laboratory analyzers. Many of the positive aspects of blood glucose self- monitoring may be traded off by spurious measurements that arise from lack of operator training, or from the monitor's inability to compensate for such biologic variables as vitamin intake and variations in hematocrit values. It is imperative that all users recognize that spurious results can occur. Therefore, clinicians who prescribe BGMs for home use should ensure that those who will operate the monitor receive adequate training.

Thus, while BGMs provide a significant advantage over urine or blood "dipstick" glucose testing, users must be aware of the limitations of these instruments.

1. Burritt MF, Klee GG, Hanson E, et al: Evaluation of the One Touch glucose meter for use by a phlebotomy team. Lab Med 1990;21:512-512.

2. Hook WC, Bell B, O'Leary J, et al: Criteria for examining the analytical performance and human engineering factors of portable blood glucose monitors. Proceedings of the 23rd Annual Meeting and Exposition of the Association for the Advancement of Medical Instrumentation, May 1988.

3. Jovanovic-Peterson L, Peterson C, Kilo C, et al: Identifying sources of error in self-monitoring of blood glucose. Diabetes Care 1988;11:791-794.

4. Kiechle FL, Ingram R, Karcher R, et al: Transfer of glucose measurements outside the laboratory. Lab Med 1990;21:504511.

5. Matthews DR, Bown E, Watson A., et al: Pen-sized digital 30-second blood glucose meter. Lancet 1987;1:778-779.

6. Shyler JS: Dietary management of diabetes mellitus, in $\mathrm{Pe}-$ terson CM (ed): Diabetes Management in the 80s: The Role of Home Blood Glucose Monitoring and New Insulin Delivery Systems. New York, Prager Scientific, 1982, pp 126-151. 